Esta revista forma parte del acervo de la Biblioteca Jurídica Virtual del Instituto de Investigaciones Jurídicas de la UNAM http://www.juridicas.unam. mx/ https://biblio.juridicas.unam.mx/bjv

https://revistas.juridicas.unam.mx/

DOI: http://dx.doi.org/10.22201/iij.24487937e.2021.15.16132 


\title{
LOS DISTINTOS TIPOS DE ANÁLISIS CONCEPTUAL Y SUS APLICACIONES EN LA TEORÍA JURÍDICA. HACIA UNA INTEGRACIÓN METODOLÓGICA*
}

\author{
THE DIFFERENT TYPES OF CONCEPTUAL ANALYSIS \\ AND ITS APPLICATIONS IN LEGAL THEORY. TOWARD \\ METODOLOGICAL INTEGRATION
}

\author{
Ángel O. Reyes FLORES**
}

\section{Resumen:}

El análisis conceptual como método en la investigación filosófica jurídica ha sido un reflejo de los desarrollos en la filosofía analítica general, dentro de estos podemos encontrar dos vertientes, la vertiente reductiva y la vertiente conectiva, así como la crítica de W. V. O. Quine en cuanto a la naturalización del análisis, las cuales se replican en la teoría jurídica; no obstante, dentro de ésta, han surgido variantes de estos enfoques en relación al concepto mismo del derecho y otros conceptos relacionados; como lo son, el análisis conceptual directamente evaluativo, o el análisis conceptual indirectamente evaluativo, así como otros enfoques que consideran que los conceptos deben analizarse en relación con sus propiedades necesarias y otros que deben analizarse en relación a sus propiedades contingentes, o bien a ambas. Asimismo, se propone que, siendo conscientes de las distinciones metodológicas del análisis conceptual, sea posible integrar metodológicamente sus vertientes.

\section{Palabras clave:}

Análisis reductivo, análisis conectivo, análisis naturalista, análisis conceptual, explicación conceptual, integración metodológica.

Artículo recibido el 24 de junio de 2019 y aceptado para su publicación el 10 de julio de 2020. Ganador del IV Concurso de Investigación en Filosofía del Derecho de Estudiantes de Posgrado: paradigmas emergentes en derecho y filosofía jurídica.

** Doctorando en el Instituto de Investigaciones Jurídicas UNAM, ORCID: https:// orcid.org/0000-0001-5825-9742, correo electrónico: oswaldo.rey@hotmail.com 


\title{
ÁNGEL O. REYES FLORES
}

\begin{abstract}
:
Conceptual analysis as a method in legal theory has been influenced by developments in general analytical philosophy, within which we can find two basic types, the reductive analysis and the connective analysis. Along with W. V. O. Quine's criticism of the naturalization of analysis, these approaches are replicated in legal theory. However, variants of these approaches have emerged around the very concept of law and other related concepts, such as the case of directly evaluative conceptual analysis and indirectly evaluative conceptual analysis, as well as other approaches claiming that concepts should be analyzed in relation to their necessary properties while others stating that said concepts should be analyzed in relation to their contingent properties, or both. It is also suggested that it is possible to methodologically integrate the different aspects of conceptual analysis while being aware of the methodological distinctions.
\end{abstract}

\section{Keywords:}

Reductive Analysis, Connective Analysis, Naturalistic Analysis, Conceptual Analysis, Methodological Integration. 
SumARIO: I. Introducción. II. Objetivo. III. Dos tipos de análisis conceptual. IV. ¿Un análisis naturalista? V. La aplicación del análisis conceptual en la teoría juridica. VI. ¿Qué tipo de concepto es el derecho? VII. Hacia una integración metodológica del análisis conceptual. VIII. Conclusiones. IX. Referencias.

\section{INTRODUCCIÓN}

En este breve estudio se pretende responder la pregunta ¿Cómo se caracteriza y justifica el análisis conceptual en la filosofía del derecho? Para ello se parte en primer lugar, de una breve descripción de los dos tipos principales de análisis que se pueden encontrar en la tradición analítica en filosofía general, así como un presunto tercer tipo de análisis que surge de la crítica de Quine sobre la revisión de proposiciones fundamentales, a la luz de evidencia empírica. Después, se exponen brevemente algunos casos de análisis conceptual en la teoría jurídica, dos casos excepcionales y dos casos paradigmáticos que se ubican en los trabajos de Jeremy Bentham y John Austin, por un lado; y Hans Kelsen y H. L. A Hart por otro, así como la propuesta de Brian Leiter de incorporar la visión naturalista en el análisis conceptual del derecho. Más tarde, se exponen algunos problemas y retos que se han suscitado con la distinción de los dos tipos de análisis en la teoría jurídica y las características del concepto del derecho, además se expone brevemente la posibilidad de integrar metodológicamente los distintos tipos de análisis para finalmente exponer las conclusiones del estudio.

\section{OBJETIVO}

Exponer como se caracteriza, aplica y justifica el análisis conceptual en la teoría jurídica, su problemática y las posibilidades de su integración metodológica. 


\section{Dos TIPOS DE ANÁLISIS CONCEPTUAL}

Para Brian Bix, el análisis conceptual en la teoría jurídica, forma parte de un enfoque en filosofía del derecho que se denomina "jurisprudencia analítica" la cual está relacionada con una disciplina más general la "filosofía analítica".

En palabras de Peter Strawson, el título de "filosofía analítica", sugiere que la descripción apropiada de su actividad favorita es el "análisis conceptual". ${ }^{2}$ De igual forma John Searle señala que "aunque no hay una división absoluta pueden identificarse dos propuestas de filosofía analítica, una que propone que el lenguaje ordinario es en general adecuado como herramienta y como materia de análisis filosófico; y otra, que propone que el lenguaje ordinario es inadecuado para propósitos filosóficos e innegablemente confuso". ${ }^{3}$

Puede reconocerse en los trabajos de Gottlob Frege, Bertrand Russell, G. Edward Moore y otros, un claro análisis conceptual; sin embargo, es en los trabajos de Wittgenstein donde encontramos descritas las bases de uno y otro tipo de análisis, de ahí la importancia de este autor para establecer las diferencias y conexiones entre ambos tipos, sobre los cuales nos referiremos en este trabajo como análisis reductivo (lógico proposicional) y al análisis conectivo (del lenguaje ordinario), distinción establecida por Peter Strawson en su obra Analysis and Methaphysics. ${ }^{4}$

El que en Wittgenstein, se reconozcan ambos tipos de análisis, muy probablemente se deba a que tuvo influencia de la tradición del positivismo lógico, que inicia con Frege y su desarrollo del lenguaje lógico simbólico perfeccionado después por Russell y Whitehead,

1 Bix, Brian, Diccionario de teoría jurídica, trad. de Enrique Rodríguez Trujano y Pedro Villarreal Lizárraga, México, UNAM-Instituto de Investigaciones Jurídicas, 2009, p. 145.

2 Strawson, Peter, Análisis y Metafísica, México, Paidós, 1992, p. 44.

3 Bunnin, Nicholas y Tsui-James, E. P., The Blackwell Companion to Philosophy, 2a edición, Estados Unidos, Blackwell Publishing company, 2003, p. 5.

4 Snowdon, Paul y Gomes, Anil, "Peter Frederick Strawson", The Stanford Encyclopedia of Philosophy (Spring 2019 Edition), Edward N. Zalta (ed.), forthcoming https://plato.stanford.edu/archives/spr2019/entries/strawson/. 
asimismo por la tradición procedente de G. E. Moore que otorgaba primacía a los juicios de sentido común y a las prácticas ordinarias para abordar los problemas filosóficos, ambas tradiciones consideran al lenguaje como el punto de partida de la filosofía; sin embargo, la primera considera que el análisis lingüístico debía hacerse mediante reducción lógica, y la segunda, el análisis del uso de los términos (conceptos) en el lenguaje ordinario. ${ }^{5}$

\section{Análisis lógico proposicional (reductivo)}

Wittgenstein escribió en el Tractatus que el planteamiento de los problemas filosóficos descansa en la incomprensión de la lógica de nuestro lenguaje. ${ }^{6}$ En esta misma publicación da algunos fundamentos para este tipo de análisis el cual considera a las proposiciones $a$ priori como las bases del pensamiento correcto al decir "un pensamiento correcto a priori sería un pensamiento tal que su posibilidad condicionaría su verdad".7 Y más adelante señala: "Sólo podríamos saber a priori que un pensamiento es verdadero si por el pensamiento mismo (sin objeto de comparación) resultara reconocible su verdad". ${ }^{8}$

Aquí podemos ver claramente el compromiso de Wittgenstein, con las verdades a priori. Asimismo, podemos ver un compromiso con el análisis lógico-proposicional al decir: "Hay un análisis completo, y sólo uno, de la proposición”. ${ }^{9}$ Es decir, el análisis versa únicamente sobre proposiciones pues "la proposición expresa de un modo determinado y claramente especificable lo que expresa: la proposición es articulada". ${ }^{10}$ Es por lo anterior, que "el nombre no

5 Nubiola, Jaime, Neopositivismo y filosofía analítica: balance de un siglo, "Filosofia nel XX secolo", Acta Philosophica, VIII/2, 1999, 197-222, disponible en: http:// www.unav.es/users/Articulo39.html.

6 Wittgenstein, Ludwig, Tractatus Logicus Philosophicus, Kegan Paul, Trench, Trubner \& Co., Ltd., Gran Bretaña, 1922, p. 5.

7 Ibidem, p. 21.

8 Idem.

9 Ibidem, p. 22.

10 Idem. 
puede ya descomponerse más por definición alguna: es un signo primitivo." Por lo tanto, "Sólo la proposición tiene sentido; sólo en la trama de la proposición tiene un nombre significado". ${ }^{11}$

Asimismo, para Wittgenstein las confusiones filosóficas se dan porque "en el lenguaje ordinario sucede con singular frecuencia que la misma palabra designe de modo y manera distintos". ${ }^{12}$ Por ejemplo: En la proposición 'Verde es verde', donde la primera palabra es el apellido de una persona y la última un adjetivo. ${ }^{13}$ La forma de eludir estos errores es usar "un lenguaje sígnico que los excluya, en la medida en que no use el mismo signo en símbolos distintos, ni use externamente de igual manera signos que designen de modo diferente. Un lenguaje sígnico, pues, que obedezca a la gramática lógica, a la sintaxis lógica." ${ }^{14}$

Las cosas que saltan a la vista en este tipo de análisis son tres, la primera que este análisis se preocupa por la proposición, no por el nombre, pues lo considera una partícula primitiva que no tiene significado sino en una proposición, en concreto, el énfasis es en el orden lógico que le da sentido a una proposición o enunciado, es decir, primero la sintaxis lógica, después la semántica. Lo segundo, que las proposiciones son analizadas en un lenguaje de signos, que menciona Wittgenstein como el lenguaje de Frege y Russell que no es otro que lo que se conoce como lógica proposicional de primer orden. Y finalmente, que las proposiciones obtenidas mediante este tipo de análisis deben ser necesariamente verdaderas a priori. ${ }^{15}$

Por tanto, este tipo de análisis consiste en obtener el significado de un concepto por medio de su reducción a una proposición elemental, eliminando sus elementos de significado mediante un lenguaje de signos, que da como resultado el poder decidir cuando una proposición es necesariamente verdadera, sin recurrir a evidencia empírica.

11 Idem.

12 Idem.

13 Ibidem, p. 27.

14 Ibidem, p. 29.

15 Idem. 


\section{Análisis lógico del lenguaje ordinario (conectivo)}

Más adelante, Wittgenstein en Investigaciones Filosóficas señala: "Reconducimos las palabras de su empleo metafísico a su empleo cotidiano. Ése es el criterio y el objetivo del método, nada más que eso, ello basta para desenmascarar todo nimbo."16

Por lo que hace al lenguaje ordinario, la condición de posibilidad de este método, sobreviene a la pregunta "bajo qué especiales circunstancias se emplea efectivamente esta oración. Es en éstas en las que tiene sentido". ${ }^{17}$ Así, el contexto es crucial para entender los planteamientos del lenguaje.

Este replanteamiento parte de que el lenguaje no es algo aislado, por lo que, debe examinarse en conjunto con la experiencia, es decir, debe perseguir determinadas finalidades prácticas, dado que "las confusiones que nos ocupan surgen, por así decirlo, cuando el lenguaje marcha en el vacío, no cuando trabaja". ${ }^{18}$

Como puede verse Wittgenstein atenúa su postura respecto a la reducción lógica de proposiciones para centrarse en el lenguaje ordinario, en concreto en lo que él llama "juegos del lenguaje" que refieren "al todo formado por el lenguaje y las acciones con las que está entretejido".19

Ahora bien, ¿en qué consiste el método del análisis conceptual en la reformulación de Wittgenstein?

\section{A. Ver lo común (encontrar parecidos de familia)}

En este sentido se debe estar atento a lo que tienen en común determinados objetos y decir esto que tienen en común se llama "X". Asimismo, afirma: "No puedo caracterizar mejor esos parecidos que con la expresión 'parecidos de familia'; pues es así como se superpo-

16 Wittgenstein, Ludwig, Tractatus logico-philosophicus-Investigaciones filosóficas-Sobre la certeza, España, Gredos, 2009, p. 261.

17 Ibidem, 263.

18 Idem.

19 Ibidem, p. 171. 
nen y entrecruzan los diversos parecidos que se dan entre los miembros de una familia: estatura, facciones, color de los ojos, andares, temperamento, etcétera, etcétera". ${ }^{20}$

\section{B. Encontrar ejemplos}

En este caso señala que hay que responderse la siguiente pregunta: “¿cómo hemos aprendido el significado de esta palabra ("bueno", por ejemplo)? ¿A partir de qué ejemplos; en qué juegos de lenguaje? Verás entonces fácilmente que la palabra ha de tener una familia de significados." ${ }^{21}$

\section{Encontrar casos normales y anormales}

Como se ha visto, el significado es dado por el uso del lenguaje. Respecto de esto señala que hay que estar alerta sobre qué es lo esencial que veamos al oír una palabra y a pesar de todo en algún caso ser distinta su aplicación. Es decir, si en algunos casos hay un empleo diferente de un término; no obstante, que en general su aplicación es una. ${ }^{22}$ Por tanto, Wittgenstein afirma "Sólo en los casos normales nos es claramente prescrito el uso de una palabra; sabemos, no tenemos duda, qué hemos de decir en este o aquel caso. Cuanto más anormal es el caso, más dudoso se vuelve lo que debemos decir entonces". ${ }^{23}$ Para ello, "no basta una definición; y mucho menos basta hacer constar que una palabra es «indefinible»". ${ }^{24}$

Con todo lo anterior, puede decirse que en este enfoque el lenguaje, no tiene esencia lógica. Es una acumulación de un gran número de diferentes "juegos de lenguaje", de los cuales el informe o la descripción de los hechos es simplemente uno. Cada uno de ellos

20 Wittgenstein, Ludwig, Tractatus logico-philosophicus-Investigaciones filosóficas... cit. p. 171.

21 Ibidem, p. 237.

22 Ibidem, p. 277-279.

23 Ibidem, p. 279.

24 Ibidem, p. 315. 
tiene su propia forma de trabajar y ya no son idénticos en lo esencial, de tal suerte que los juegos ordinarios, se relacionan entre sí, sólo por "semejanza familiar". ${ }^{25}$

En este sistema, el significado de una palabra u oración se encuentra en las reglas de su uso en la vida real, no en la reflexión filosófica. Estas reglas se distinguen mejor en la actividad de aprender a usar las expresiones involucradas; son el resultado de convenciones que puede ser alterado; pero estas convenciones deben ser públicas y compartidas, por tanto, un lenguaje privado es imposible. ${ }^{26}$

\section{IV. ¿UN ANÁLISIS NATURALISTA?}

Como se ha visto uno de los supuestos, por lo menos del análisis reductivo es la noción de que una proposición analítica es inmune a su revisión, puesto que es necesariamente verdadera. En este respecto, el trabajo de Willard Van Orman Quine es referente, debido a que argumentó que no hay proposiciones inmunes a su revisión, que cada proposición puede ser revisada con base en evidencia empírica. $^{27}$

De acuerdo con Quine, "El empirismo moderno ha sido en gran parte condicionado por dos dogmas. Uno de ellos es la creencia en cierta distinción fundamental entre verdades que son analíticas, basadas en significaciones, con independencia de consideraciones fácticas, y verdades que son sintéticas, basadas en los hechos. El otro dogma es el reductivismo, la creencia en que todo enunciado que tenga sentido es equivalente a alguna construcción lógica basada en términos que refieren a la experiencia inmediata". ${ }^{28}$

Lo anterior, supone la formulación de enunciados no contradictorios, a partir de los hechos que instancian una categoría conceptual dada, lo cual permite hacer ajustes en la estructura de conceptos que

25 Honderich, Ted, The Oxford Companion to Philosophy, Estados Unidos, Oxford University Press, 1995, p. 490.

26 Ídem.

27 Bunnin, Nicholas y Tsui-James, E. P., op cit., p. 6.

28 Quine, Willard Van Orman, "Dos dogmas del empirismo", La búsqueda del significado, Universidad de Murcia, España, 1991, p. 49. 
eran dados por verdaderos a priori. Algunos suponen que, si esto pasa, los resultados del análisis filosófico no pueden ser distintos de los resultados de las investigaciones científicas. ${ }^{29}$ No obstante, lo anterior, sería una apreciación bastante extrema.

Como ya se ha mencionado, la crítica naturalista fue dirigida principalmente al reductivismo, por lo que parece ser que el análisis conectivo puede permanecer incólume ante tales aspectos, pues al considerar casos normales y casos anormales, puede decirse que no se aparta totalmente de los hechos.

Aún el análisis reductivo, tampoco parece afectarse con esta distinción a menos que las proposiciones formuladas por reducción no refieran ninguna experiencia posible. Como señala Peter Strawson, "los conceptos de lo real no pueden significar nada para sus usuarios a no ser que se relacionen, directa o indirectamente, con una posible experiencia de lo real". ${ }^{30}$

Tal vez, una forma caritativa y menos extrema de interpretar la crítica quineana sea que la filosofía deba apoyarse y apoyar a las ciencias empíricas, como una disciplina general y crítica del conocimiento obtenido por los métodos de dichas ciencias, pero no constituirse como una ciencia más.

\section{LA APLICACIÓN DEL ANÁLISIS CONCEPTUAL EN LA TEORÍA JURÍDICA}

La visión más extendida sobre la filosofía analítica en la teoría jurídica es que ésta ofrece analizar la naturaleza básica del derecho y los conceptos jurídicos. ${ }^{31}$ En atención a lo anterior, es procedente hacer la siguiente pregunta: ¿Es aplicable a la teoría jurídica la distinción hecha en el punto anterior? La respuesta es que sí, puesto que el método de análisis en sus variantes descritas líneas arriba es empleado

29 Bunnin, Nicholas y Tsui-James, E. P., op cit., p. 7.

30 Strawson, Peter, op. cit., p. 101.

31 Bix, Brian, "Some reflections on methodology in jurisprudence", Problemas contemporáneos de la filosofía del derecho, México, UNAM, Instituto de Investigaciones Jurídicas, 2005, pp. 67-96. 
LOS DISTINTOS TIPOS DE ANÁLISIS CONCEPTUAL...

en la teoría jurídica, con algunos casos excepcionales y algunos problemas propios de la disciplina que se verán a continuación. ${ }^{32}$

\section{Casos excepcionales. Jeremy Bentham y John Austin}

Como es sabido en la teoría jurídica los casos de Jeremy Bentham y John Austin, son reconocidos como exponentes de la Analytical Legal Theory; no obstante, ellos no utilizan propiamente el método descrito líneas arriba, en ninguna de sus variantes, debido a que, los trabajos de los precursores de la filosofía analítica como sería el caso de Frege, Russell y Wittgenstein surgen a finales del siglo XIX y principios del XX, y los trabajos de Jeremy Bentham y John Austin en el siglo XVIII y XIX respectivamente. No obstante, debe existir una razón por la cual se consideran exponentes de la "teoría legal analítica", lo cual, como se vio arriba, no es lo mismo que "jurisprudencia analítica", ni "filosofía analítica del derecho".

La respuesta para algunos teóricos como Endicott, es que para Bentham, la forma en la que se manifiesta empíricamente el derecho es a través de actos lingüísticos, por lo tanto, al estilo del análisis conceptual, él basa su teoría en el significado y uso de las palabras. ${ }^{33}$

32 No obstante, para Bix hay una distinción entre "jurisprudencia analítica" que es "Un enfoque de la filosofía del derecho que enfatiza el análisis de los conceptos ('sistema jurídico', 'derecho', 'propiedad'). Este enfoque está relacionado con una disciplina más general, la 'filosofía analítica'”. Y “filosofía analítica del derecho” 'que supone (del derecho) es una búsqueda de los significados de términos y conceptos. Los filósofos analíticos tienden a creer que el análisis conceptual es un enfoque defendible para comprender partes de nuestro mundo'. Véase, “Jurisprudencia analítica”, Bix, Brian H., Diccionario de teoría jurídica, trad. de Enrique Rodríguez Trujano y Pedro Villarreal Lizárraga, México, UNAM-Instituto de Investigaciones Jurídicas, 2009, p. 145. Asimismo, para algunos como Riccardo Guastini "la filosofía analítica del derecho positivo no es otra cosa que el análisis lógico del lenguaje jurídico... en la práctica jurídica el lenguaje del legislador se encuentra en una continua relación osmótica con el lenguaje de los juristas, sobre todo jueces y juristas académicos... Por lo tanto, hace falta entender (o extender) el análisis sugerido por Bobbio más allá del lenguaje "del legislador", hasta incluir el lenguaje de los operadores jurídicos en general." Véase, Guastini, Riccardo, Las dos caras de la filosofía analítica del derecho positivo, Italia, Istituto Tarello per la Filosofia del diritto, 2016, p. 3.

33 Endicott, Timothy, "Law and Language", The Stanford Encyclopedia of Philo- 
Además, Bentham parece pensar en el significado de una palabra en términos causales, como su capacidad de actuar en un sujeto, emergiendo una imagen de sustancias perceptibles o emociones a las cuales la palabra nombra, en estos términos generales o nombres, cosas, personas, actos, etcétera, son traídos a la vista. Por lo tanto, las palabras que no traen una vista de cosas perceptibles, no tienen significado, excepto aquellas que pueden ser expuestas por paráfrasis, o sea por el método de trasladar enunciados completos a otros en donde las palabras son usadas en enunciados de los que surgen imágenes de cosas perceptibles. ${ }^{34}$

Con este método de análisis Bentham define a la ley como un "ensamblaje de signos declarativos de una voluntad concebida o adoptada por el soberano en un Estado, que se ocupa de que una conducta sea observada en cierto caso por cierta persona o clase de personas, quienes en el caso en cuestión son o se supone que deben ser sujetos a este poder". ${ }^{35}$ Con esto sentaría las bases de un análisis de conceptos sobre el eje de la voluntad con los cuales construyó un sistema lógico muy familiar a lo que hoy conocemos como lógica deóntica y que llamó "lógica de la voluntad" o "lógica del imperativo". ${ }^{36}$

A una conclusión similar arriba J. J. Moreso quien afirma, "la filosofía de Bentham se ancla en el empirismo inglés y algunas de sus ideas prefiguran algunos de los desarrollos posteriores de la filosofía del lenguaje". ${ }^{37}$

De igual forma ocurre con John Austin quien en su estudio The Province of jurisprudence Determined señala como uno de sus objetivos determinar implícitamente la esencia o naturaleza de una orden (command) y distinguir ordenes que son leyes o reglas de órdenes que son meramente ocasionales o particulares. Determinar la naturaleza de una orden, fijar los significados de los términos que el tér-

sophy (Summer 2016 Edition), Edward N. Zalta (ed.), disponible en: https://plato. stanford.edu/archives/sum2016/entries/law-language/

34 Idem.

35 Idem.

36 Idem.

37 Moreso, Josep, Positivismo Jurídico y Filosofía Analítica, Teoría y derecho: revista de pensamiento jurídico, núm. 22, 2017, pp. 118-136 
mino orden implica: denominados "sanción" u "obediencia forzada"; "deber" u "obligación"; "superior e inferior". ${ }^{38}$ De esta forma, Austin caracteriza al derecho como ordenes respaldadas por sanciones, emitidas por un soberano que es obedecido habitualmente, lo cual lo acerca bastante a un análisis reductivo.

\section{Dos casos paradigmáticos de análisis reductivo y conectivo en la teoría jurídica}

\section{A. Hans Kelsen}

De igual forma que Jeremy Bentham, en Hans Kelsen, partiendo del concepto de grundnörm, se dan las bases para una lógica mediante la cual sea posible determinar las normas de un sistema jurídico, como objeto de estudio de una ciencia jurídica. Para conseguir tal objetivo, parte de un sistema lógico, como más adelante lo especificaría en el ensayo Lógica y Derecho, que establece un principio de inferencia y otro de contradicción. ${ }^{39}$

De acuerdo con Kelsen una norma (enunciado normativo) no puede tener como fundamento de validez la autoridad, sino otra norma (Hay que recordar que Kelsen al igual que Bentham, busca desmitificar la norma jurídica de entidades metafísicas o sobrehumanas como Dios o El Rey y darles un fundamento racional). Esta norma que refiere Kelsen no es una norma impuesta por ninguna autoridad, es más bien presupuesta, es la norma fundante básica denominada gründnorm. Esta norma presupuesta establece, de acuerdo con el autor de la Teoría Pura, la forma en la que deben crearse las normas del sistema jurídico, en este sentido señala: "El fundamento de validez de una norma solo puede encontrarse en la validez de otra norma. La norma que representa el fundamento de

38 Austin, John, The Province of Jurisprudence Determined, Reino Unido, Cambridge University Press, 1995, p. III.

39 Ordoñez, Ulises, “Consideraciones sobre 'Derecho y lógica' de Hans Kelsen”, Crítica: Revista Hispanoamericana de Filosofia, núm 10, 1978, pp. 55-79. 
validez de otra es caracterizada, metafóricamente, como una norma superior en relación con otra inferior". ${ }^{40}$

Con lo anterior, Kelsen da los fundamentos para el análisis de conceptos jurídicos los cuales se reducen a ciertos enunciados de los cuales parecen desprenderse las posibilidades de un sistema jurídico en términos lógicos deductivos. Aquí es donde se encuentra la regla de inferencia que remite la validez de una norma a la gründnorm, lo cual determina el concepto de sistema normativo, pues Kelsen afirma que "todas las normas cuya validez pueda remitirse a una y misma norma fundante básica constituyen un sistema de normas, un orden normativo.. ${ }^{41}$ Esto es la base de su teoría, la cual, hay que señalar que vale solo para las normas creadas bajo este esquema, y en donde Kelsen ve una descripción de un sistema positivista. ${ }^{42}$

De igual forma una vez que se tiene esta regla general que valida las normas del sistema, Kelsen establece un principio que deben cubrir todas aquellas normas descritas en enunciados normativos para que el sistema sea coherente, lo cual establece en estos términos: "Puesto que la norma fundante básica es el fundamento de validez de todas las normas pertenecientes a un mismo orden jurídico, constituye ella la unidad dentro de la multiplicidad de esas normas, esa unidad también se expresa diciendo que el orden jurídico es descrito en enunciados jurídicos que no se contradicen". ${ }^{3}$

De acuerdo a las reglas de su sistema, Kelsen plantea que los conflictos se pueden dar de diversas formas, se plantea el problema del conflicto entre normas del mismo nivel o de un conflicto entre una norma de nivel superior y una norma de nivel inferior, o bien las emitidas por un mismo órgano o por órganos diferentes, o simplemente cuando la ley contiene palabras sin sentido o disposiciones entre si incompatibles, o bien puede aparecer también un conflicto entre dos normas individuales cómo pueden ser dos sentencias judiciales especialmente cuando ambas normas han sido dictadas por órganos

40 Kelsen, Hans, Teoría Pura del Derecho, trad., Roberto J. Vernengo, México, UNAM, 1979, p. 201.

41 Ibidem, p. 202.

42 Ibidem, p. 214.

43 Idem. 
diferentes. ${ }^{44}$ De acuerdo con lo anterior, por lo que hace a esta parte de su estudio puede considerarse como un reduccionista. ${ }^{45}$

\section{B. H. L. A Hart}

Hart desde el principio de su obra The Concept of Law, señala que su objetivo es "promover la comprensión del derecho, la coerción y la moral, en cuanto fenómenos sociales diferentes, aunque relacionados". ${ }^{46}$ Hay que señalar que la reflexión sobre el concepto

44 Ibidem, pp. 215-217.

45 Si bien, es controversial la influencia de la filosofía analítica en Kelsen el cual se considera como un neo-kantiano, no puede negarse que estaba al tanto de algunos desarrollos en filosofía analítica como los de George Edward Moore, a quien cita "Con respecto al concepto de "deber" vale lo mismo que George Edward Moore (Princípia Ethica, Cambridge, 1922, pp. 7 y ss.) dice del concepto 'bueno'... 'bueno' es una noción simple, así como 'amarillo' es una noción simple”. Un concepto simple no es definible y lo que conduce a lo mismo, no es analizable... El contenido del deber consiste en aquello que un orden positivo, moral o jurídico, prescribe; es determinado a través de actos de voluntad y cuando es así determinado, es reconocido". Véase, Kelsen, op. cit., p. 19. De igual forma, no era ajeno al análisis de conceptos pues en una referencia al concepto de "regla" de Moritz Schlick señala "El concepto no expresa que algo debe contar con las propiedades que la definición determina. El concepto de una buena conducta es: conducta que corresponde a una norma. Este concepto contiene tres elementos: 'norma', 'conducta' y 'correspondencia' en cuanto relación entre 'norma' y 'conducta'. Este concepto no expresa que una conducta deba corresponder a una norma, sino solamente que, cuando no corresponde a una norma, no encaja en el concepto de buena conducta y, por ende, que no es una conducta buena. Que la conducta deba corresponder a la norma, es el sentido de la 'norma', que junto con 'conducta' y 'correspondencia', configura un elemento del concepto de buena conducta, pero no el sentido del concepto. La conducta es buena, no por corresponder al concepto, sino por corresponder a la norma. Puede contradecir la norma, pero no al concepto". Véase, Kelsen, op. cit., p. 31. Para Vernengo, Kelsen llega a la misma conclusión que Von Wright, al escribir en la segunda edición de la Reine Rechtslehre que "'los principios lógicos pueden ser aplicados a normas jurídicas, si bien no directamente, por lo menos indirectamente, en cuanto son aplicables a las proposiciones jurídicas que describen a esas normas'." Véase, Vernengo, Roberto, Derecho y lógica: un balance provisorio, Anuario de filosofía del derecho, no 4, 1987, pp. 303-330.

46 Hart, H., El concepto de derecho, trad. de Genaro Carrió, Argentina, AbeledoPerrot, 1961, p. XI. 
del derecho alude directamente al trabajo de John Austin en The Province of Jurisprudence Determined, quien como ya se ha comentado, ofrece una caracterización reductiva del derecho como "ordenes respaldadas por amenazas", la cual está basada en el concepto de la ley como mandato de Jeremy Bentham. Por lo que, la tarea de Hart, en la primera parte de su obra, es señalar los problemas que esta caracterización entraña, así como ofrecer una caracterización compatible con otras formas de acciones consideradas comúnmente como jurídicas.

Hart inicia su estudio con el problema que representa la pregunta sobre "el derecho", referente a este tema señala: "Pocas preguntas referentes a la sociedad humana han sido formuladas con tanta persistencia y respondidas por pensadores serios de maneras tan diversas, extrañas, y aun paradójicas, como la pregunta ¿qué es derecho?”.

Respecto de este problema, duda si se está realizando la pregunta correcta, pues el derecho es un concepto problemático debido a su similitud con otros conceptos como los de la religión y la moral. Asimismo, menciona que, si bien, existen casos típicos de lo jurídico, también existen casos, en los cuales la calidad de ser jurídicos no está completamente determinada, por lo que puede haber dudas aún para los más documentados en la materia.

Señala como uno de esos casos problemáticos, el del derecho internacional, pues a diferencia de otros, éste no es creado por ninguna legislatura y los estados no se someten a una jurisdicción internacional, debido a que las Cortes Internacionales no tienen facultades para hacer cumplir sus determinaciones, además de que sus sanciones son indeterminadas. De igual forma, señala al derecho primitivo, que comparte algunas similitudes con el derecho internacional.

Es claro que en esta parte de su estudio Hart está siguiendo algunas de las reglas del método del segundo Wittgenstein, buscando parecidos de familia, casos normales y casos anormales.

\section{La propuesta naturalista en la teoría jurídica}

Una propuesta que se ha manifestado es la de convertir a la filosofía del derecho en una disciplina naturalizada, en el sentido de la 
crítica quineana sobre el análisis conceptual, el esfuerzo más claro por lograr este objetivo, tal vez, sea el de Brian Leiter, quien señala que el análisis conceptual es preferido en la jurisprudencia porque facilita el éxito de teorías a posteriori sobre el derecho y las instituciones legales. Asimismo, señala que la única forma de reivindicar los argumentos conceptuales del positivismo duro, es que el concepto que mejor explica es el que se encuentra en los programas de investigación a posteriori, esto es, aquellos que proporcionen un entendimiento adecuado de cómo funciona el mundo. ${ }^{47}$

Sugiere tomar en cuenta la visión del derecho que los antropólogos, sociólogos, psicólogos y otros puedan decirnos de las prácticas sociales sobre el derecho. ${ }^{48}$ Como ejemplos de este tipo de programas señala a aquellos que en el espíritu del realismo entienden la operación de las cortes en términos socio-demográficos para explicar su comportamiento, los cuales han asumido que las explicaciones basadas en el derecho están confinadas a explicaciones en términos de normas con pedigrí ${ }^{49}$ De igual forma menciona que estos científicos sociales nos proporcionan una visión de las cortes que encaja en una más amplia concepción naturalista del mundo, con causas y reglas deterministas donde la voluntad o la agencia no juegan ningún rol explicativo. ${ }^{50}$

Asimismo, propone voltear la mirada al realismo como un programa de investigación de carácter predictivo sobre cómo decidirán los jueces, así como su compromiso con el naturalismo y el pragmatismo. ${ }^{51}$

No obstante, para algunos teóricos del análisis conceptual tradicional como Kenneth Himma, nadie ha articulado una declaración

47 Leiter, Brian, "Legal Realism, Hard Positivism, and the Limits of Conceptual Analysis", en Coleman, Jules, Hart's Postscript Essays on the Poscript to The Concept of Law, Oxford University Press, 2001, p. 369.

48 Idem.

49 Ibidem, p. 370.

50 Idem.

51 Leiter, Brian, Naturalismo y teoría del derecho, trad. de and Giovanni Battista Ratti, España, Marcial Pons, 2012, p. 56 
razonablemente clara de cómo debe proceder una jurisprudencia naturalizada con respecto al análisis de conceptos. ${ }^{52}$

Quizás la descripción de una teoría naturalizada corresponde a lo que se conoce como el movimiento "Law and Society" que se describe como los esfuerzos de los sociólogos del derecho, antropólogos del derecho, politólogos que estudian el comportamiento judicial, historiadores que exploran el rol de los abogados del siglo XIX, psicólogos que se preguntan sobre cómo se comportan los jurados, etc. ${ }^{53}$

\section{VI. ¿QUÉ TIPO DE CONCEPTO ES EL DERECHO?}

Un par de nuevos enfoques sobre el análisis conceptual y que tienen que ver con el concepto mismo del derecho, son el enfoque directamente evaluativo y el enfoque indirectamente evaluativo, cuyos exponentes más connotados son Ronald Dworkin y Julie Dickson, respectivamente.

Para Ronald Dworkin, el concepto del derecho es en parte evaluativo y requiere una metodología evaluativa para explicarlo. ${ }^{54}$ Es decir, el derecho es un concepto interpretativo que se basa en ciertas normas substantivas, su teoría está fundada en la interpretación constructiva de las acciones oficiales. ("Interpretación constructiva" es el principio que Dworkin habría aplicado no sólo para resolver disputas jurídicas, sino también para construir teorías sobre la naturaleza del derecho.) Así por "derecho", Dworkin entendería "el hecho de que los jueces decidiesen los casos a partir de una teoría de las decisiones anteriores de los funcionarios (la legislación, la constitución, y las decisiones judiciales) relacionadas con la disputa que se ajustase adecuadamente a aquellas decisiones del pasado y pre-

52 Himma, Kenneth, “Conceptual Jurisprudence An Introduction to Conceptual Analysis and Methodology in Legal Theory”, Revus, núm 26, 2015, pp. 65-92.

53 Friedman, Lawrence M., The Law and Society Movement, Stanford Law Review, vol. 38, núm. 3, 1986, pp. 763-780.

54 Himma, Kenneth, op. cit. p. 80. 
sentase a esa área del derecho lo mejor que sea posible (moral o políticamente)". ${ }^{55}$

Por otro lado, Julie Dickson, sostiene que es posible realizar evaluaciones que no refieran a la moralidad de las normas en el derecho, a esto le llama "evaluación indirecta", la cual consiste en evaluar cuales son las propiedades relevantes de un concepto y distinguirlas de las no relevantes; sin apelar a la "evaluación directa", en el sentido de que una evaluación de este tipo, requiere el recurso a las normas morales que establecen qué contenido las prácticas sociales relevantes deberían tener. ${ }^{56}$

Respecto de este problema, algunos teóricos como Brian Bix se han planteado ¿si el derecho es un concepto con propiedades necesarias? Para ello propone, en primera instancia, distinguir la aplicación a los términos que refieren entidades físicas o naturales, y por otro lado, su aplicación en instituciones humanas y prácticas sociales, los cuales parecen ser mucho más problemáticos. ${ }^{57}$

A este respecto señala: "La discusión jurídica sobre la naturaleza del derecho no es un análisis de necesidad lógica o un tipo natural. Es un análisis conceptual, y lo que sea necesario o esencial está envuelto en una investigación de conceptos". ${ }^{58}$ En este respecto la respuesta apropiada es que el análisis conceptual es una investigación a priori, es decir, no podemos estudiar el derecho hasta que sabemos que significamos como "derecho"..$^{59}$ Asimismo, como ejemplo de la distinción entre características necesarias y contingentes menciona a Joseph Raz al decir:

Raz similarmente escribe sobre el concepto un derecho que parece ser ambos, contingente y necesario (o, en su propia terminología, ambos "parcial" y "universal”). De acuerdo con Raz: (1) Tenemos un concepto de derecho; (2) basado en cómo se entiende nuestra

55 Bix Brian, op. cit., nota 1, pp. 63 y 64.

56 Himma Kenneth, p. 82.

57 Bix, Brian, Some reflections on methodology in jurisprudence, Problemas contemporáneos de la filosofía del derecho, México, UNAM, Instituto de Investigaciones Jurídicas, 2005, pp. 70 y 71

58 Ibidem, p. 71.

59 Ibidem, p. 72. 
sociedad a sí misma y (3) nuestro concepto de derecho ha cambiado a través del tiempo, en respuesta a los cambios en las instituciones, prácticas, actitudes, y aún las teorías filosóficas. ${ }^{60}$

Para Bix, lo anterior, "es la justificación del análisis conceptual en la teoría jurídica, pero no solo el concepto de derecho necesita ser explicado, antes de entrar a cualquier tipo de validación empírica, también lo serían otros como obligación, deber y regla con los cuales está emparentado". ${ }^{61}$

Asimismo, Bix plantea que para aquellos que sostienen que el derecho es un concepto con parecidos familiares, es decir, con instanciaciones que no tienen característica en común y de ahí que no tenga características necesarias, la forma de "refutar" esta creencia es proveer un análisis en términos de condiciones necesarias y suficientes, como Raz y otros han intentado hacer. ${ }^{62} \mathrm{Si}$ el análisis es exitoso, esto es suficiente para mostrar que el "derecho" no es un concepto con parecido familiar. ${ }^{63}$

Así este debate está lejos de ser terminado, ya que, algunos como Michael Giudice, señalan que las características que pueden ser más relevantes del concepto del derecho, no son las necesarias sino las contingentes, lo cual favorece la comprensión del derecho en otros contextos y en conexión con otros fenómenos. Respecto de esto señala:

Mi tesis es que una explicación analítica conceptual del derecho puede y debe reconocer la importancia de las características contingentes y las relaciones críticas, para una explicación más profunda de las varias experiencias sobre el derecho en diversas comunidades. Una vez entendida de esta manera, la jurisprudencia analítica puede apuntar nuevamente a verdades universales sobre la naturaleza de derecho, compuesto de una combinación de características necesarias y contingentes y sus relaciones. Tal comprensión renovada conserva el objetivo histórico de la jurisprudencia analítica para proporcionar una explicación universal del derecho al tiempo que se reconoce la multifacética naturaleza del derecho

$$
\begin{aligned}
& \text { Problema. Anuario de Filosofia y Teoría del Derecho } \\
& \text { Núm. 15, enero-diciembre de 2021, pp. 549-574 }
\end{aligned}
$$


(y su explicación conceptual) como un reflejo de muchas y muy variadas situaciones sociales. ${ }^{64}$

\section{HACIA UNA INTEGRACIÓN METODOLÓGICA DEL ANÁLISIS CONCEPTUAL}

A lo largo de esta reflexión, se ha caracterizado al análisis conceptual en la teoría jurídica, como una extensión de las dos formas, reductiva y conectiva desarrolladas en la filosofía general, así como el reto que plantea la crítica quineana al análisis sobre la reducción lógica y las verdades a priori.

Estas visiones del análisis plantean; por un lado, el problema de si es posible reducir un concepto como el derecho a características necesarias y suficientes, además de cuáles son las características relevantes del concepto, como ya se vio arriba; y por otro lado, si realmente un concepto como el derecho puede aspirar a ser inmune a la contrastación empírica, estos retos son los retos internos del análisis conceptual, además de un tercer reto que viene desde fuera del análisis conceptual, la necesidad de un concepto universal del derecho ante un contexto de pluralismo legal.

Estos retos son planteados por Michael Giudice, en cuanto al primero señala, que el análisis conceptual del derecho, para despegar, debe presumir que hay un solo, concepto coherente y unificado del derecho que subyace a las diversas intuiciones y usos lo cual parece bastante difícil. ${ }^{65}$ Por lo que hace al segundo, establece la posibilidad de acuerdo con Leiter, de proporcionar una forma de examinar como algunos conceptos de derecho son superiores a otros, sin apelar a las intuiciones o algún otro pseudo-método empírico. ${ }^{66}$

En cuanto al tercer reto menciona, "el pluralismo legal es una especie de teoría socio-legal dedicada a la exploración de una amplia variedad de derechos alrededor del mundo, y la jurisprudencia ana-

64 Giudice, Michael, Understanding the Nature of Law. A case for constructive conceptual explanation, Reino Unido, Elgar Studies in Legal Theory, 2015, p. 5.

65 Giudice, Michael, op. cit., pp. 26 y 27.

66 Ibidem, p. 28. 
lítica por otro lado, está simplemente dedicada a las cuestiones a priori sobre la naturaleza del autoconocimiento del derecho. ¿Cómo podría haber algún conflicto significativo entre estos proyectos radicalmente diferentes?"67

Asimismo, Giudice propone ir más allá del análisis hacia la explicación constructiva conceptual, entendida como la construcción filosófica de conceptos nuevos y mejorados para ser usados en la explicación y el entendimiento de la realidad social. ${ }^{68}$

Desde mi punto de vista, estos retos que plantea el análisis conceptual en la teoría jurídica pueden afrontarse de una forma diferente, lo que se propone en el presente estudio, es la posibilidad de integrar los distintos elementos del análisis conceptual en un método que puede servir a distintos objetivos.

El primer paso, como se ha pretendido en este breve estudio, es hacer explícitas las diferencias metodológicas en el análisis conceptual del derecho, y entender los alcances de cada una. Un segundo paso, consiste en estudiar con qué objetivos se ha utilizado el análisis conceptual y cuales han sido los avances realizados. Finalmente, la integración del análisis como herramienta de investigación filosófica y coadyuvante de la investigación empírica.

En cuanto al primer paso, considero que cada tipo de análisis puede aportarnos algo diferente, así el análisis reductivo nos permite juzgar más fácilmente cuales son los casos claros de un concepto, mientras que el análisis conectivo, nos permite contrastar casos claros con casos difíciles, poniendo a prueba los límites de un concepto, mientras que el análisis naturalista, nos permite encontrar nuevas propiedades o establecer las relaciones causales implícitas en los conceptos con ayuda de los métodos y datos aportados por otras ciencias.

En cuanto al segundo paso, cabría hacer un estudio de los debates y críticas que se han hecho sobre el análisis; por ejemplo, el debate Hart-Dworkin, que sugiere por un lado que Hart mediante su análisis estaba en busca del significado de la palabra "Derecho" lo cual ha sido desmentido por algunos como Joseph Raz, sugiriendo que los

67 Ibidem, p. 32.

68 Ibidem, p. 38. 
objetivos de Hart eran diferentes, al decir, “Dworkin veía el análisis conceptual en un nivel práctico como forma para justificar argumentativamente una decisión... Hart señala que la teoría que propone en The Concept of Law es una teoría explicativa sobre la regla de reconocimiento en todos los sistemas legales. En otras palabras, Hart buscaba una interpretación de la compleja institución que llamamos derecho." ${ }^{69}$ Es decir, Hart utilizó su análisis para entender al derecho y algunas prácticas relacionadas y no buscaba el significado de la palabra "derecho", es decir, el malentendido es que Dworkin veía el análisis con objetivos diferentes.

Finalmente, un ejemplo de la posibilidad de este proyecto es el trabajo de H. L. A. Hart quien realiza una reducción conceptual para caracterizar a un sistema jurídico como la unión de reglas primarias y secundarias, pero por otro lado, realiza una conexión conceptual, a partir de ejemplos, para explicar que frente a ese caso claro, existen otros sistemas que no son claros como el derecho internacional; o bien con base en su reducción del concepto de sistema jurídico como unión de reglas primarias y secundarias, construye el concepto de regla de reconocimiento, el cual puede ser puesto a prueba por algún experimento social y ser revisado.

\section{CONCLUSIONES}

Reconocer los distintos tipos de análisis conceptual en la teoría jurídica, lejos de debilitar la visión analítica, la fortalece y obliga a cuestionarse, si esas diferencias en el método no son más bien, diferencias de objetivos, y si realmente el análisis reductivo no puede coexistir con el conectivo, o de igual forma, la crítica quineana destruye por completo el análisis, o si realmente la explicación constructiva está desvinculada de la reducción y la conexión de conceptos. Desde mi punto de vista, si bien es cierto el análisis conceptual tiene una raíz reductiva, también lo es que actualmente, difícilmente

69 Raz, Joseph, "Two Views of the Nature of the Theory of Law: A Partial Comparison", en Coleman, Jules, Hart's Postscript Essays on the Poscript to The Concept of Law, Oxford University Press, 2001, p. 1. 
se podría sostener que los distintos exponentes del análisis conceptual son únicamente reduccionistas o conectivistas, ejercicios como este trabajo, sugieren que quizás las críticas no han tenido como blanco el análisis, sino los objetivos para los que se ha empleado, por ejemplo, para llegar a conceptos universales, conceptos con un contenido moral, etc. Asimismo, la integración metodológica del análisis conceptual, puede enriquecer nuestro conocimiento del derecho y las prácticas asociadas a él.

\section{REFERENCIAS}

Austin, John, The Province of Jurisprudence Determined, Reino Unido, Cambridge University Press, 1995.

BIX, Brian, "Some Reflections on Methodology in Jurisprudence", Problemas contemporáneos de la filosofía del derecho, Instituto de Investigaciones Jurídicas de la UNAM, México, 2005.

BIX, Brian, Diccionario de teoría jurídica, trad. de Enrique Rodríguez Trujano y Pedro Villarreal Lizárraga, México, UNAM, Instituto de Investigaciones Jurídicas, 2009.

Bunnin, Nicholas y Tsui-James, E. P., The Blackwell Companion to Philosophy, 2a edición, Estados Unidos, Blackwell Publishing company, 2003.

EndicotT, Timothy, "Law and Language", The Stanford Encyclopedia of Philosophy (Summer 2016 Edition), Edward N. Zalta (ed.), disponible en: https://plato.stanford.edu/archives/sum2016/entries/law-language/.

Friedman, Lawrence M., "The Law and Society Movement", Stanford Law Review, vol. 38, núm. 3, 1986.

GIUDICE, Michael, Understanding the Nature of Law A case for constructive conceptual explanation, Reino Unido, Elgar Studies in Legal Theory, 2015.

Guastini, Riccardo, Las dos caras de la filosofía analítica del derecho positivo, Italia, Istituto Tarello per la Filosofia del diritto, 2016. 
HART, H., El concepto de derecho, trad. de Genaro Carrió, Argentina, Abeledo-Perrot, 1961.

Himma, Kenneth, "Conceptual Jurisprudence An Introduction to Conceptual Analysis and Methodology in Legal Theory", Revus, núm 26, 2015, pp. 65-92.

Honderich, Ted, The Oxford Companion to Philosophy, Estados Unidos, Oxford University Press, 1995, p. 490.

Kelsen, Hans, Teoría pura del derecho, trad. de Roberto J. Vernengo, México, UNAM, 1979.

Leiter, Brian, "Legal Realism, Hard Positivism, and the Limits of Conceptual Analysis", en Coleman, Jules, Hart's Postscript Essays on the Poscript to The Concept of Law, Oxford University Press, 2001.

LeITER, Brian, Naturalismo y teoría del derecho, trad. de and Giovanni Battista Ratti, España, Marcial Pons, 2012.

Moreso, Josep, Positivismo Jurídico y Filosofía Analítica, Teoría y derecho: revista de pensamiento jurídico, núm. 22, 2017.

Nubiola, Jaime, "Neopositivismo y filosofía analítica: balance de un siglo", Filosofia nel XX secolo, Acta Philosophica, VIII/2, 1999, 197-222, disponible en: http://www.unav.es/users/Articulo39.html.

ORDoÑEz, Ulises, “Consideraciones sobre 'Derecho y lógica' de Hans Kelsen”, Crítica: Revista Hispanoamericana de Filosofia, núm. 10, 1978.

Quine, Willard Van Orman. "Dos dogmas del empirismo”, La búsqueda del significado, Universidad de Murcia, España, 1991.

RAZ, Joseph, "Two Views of the Nature of the Theory of Law: A Partial Comparison", en Coleman, Jules, Hart's Postscript Essays on the Poscript to The Concept of Law, Oxford University Press, 2001.

Snowdon, Paul y Gomes, Anil, "Peter Frederick Strawson”, The Stanford Encyclopedia of Philosophy (Spring 2019 Edition), Edward N. Zalta (ed.), disponible en: https://plato.stanford. edu/archives/spr2019/entries/strawson/. 


\section{ÁNGEL O. REYES FLORES}

STrawson, Peter, Análisis y metafísica, México, Paidós, 1992.

Vernengo, Roberto, "Derecho y lógica: un balance provisorio", Anuario de filosofía del derecho, núm. 4, 1987.

WitTgenstein, Ludwig, Tractatus logico-philosophicus-Investigaciones filosóficas-Sobre la certeza, España, Gredos, 2009.

Wittgenstein, Ludwig, Tractatus Logicus Philosophicus, Gran Bretaña, Kegan Paul, Trench, Trubner \& Co., Ltd., 1922. 